\section{REPRODUCTIVE HEALTH}

\section{A monthly oral contraceptive}

Sci. Transl. Med. 11, eaay2602 (2019)

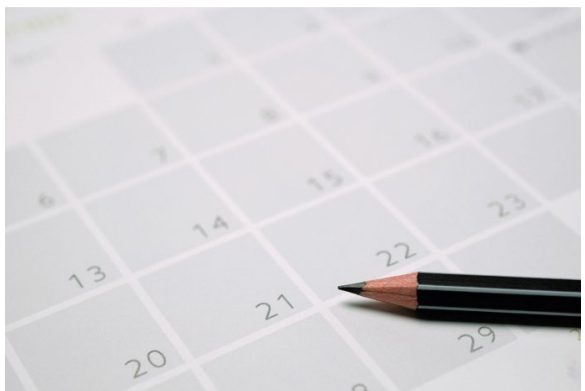

Credit: Nv Phngs Caeng Kml Kul Chay/ EyeEm/Getty

An oral contraceptive that is administered in a gastric-resident dosage form can be taken monthly to increase the ease of patient adherence.

The ability to choose to avoid unplanned pregnancies confers numerous health benefits and immeasurable social and economic advantages to those who can become pregnant. Oral contraceptives currently need to be taken daily, and the biggest cause of failure is poor patient adherence.

Ameya Kirtane and colleagues developed an oral contraceptive that is able to provide month-long doses of the active ingredient, levonestrogel, as it becomes lodged in the entrance to the stomach. They tested it in pigs and found that it delivered the active ingredient as would be required for contraceptive purposes, showing promise for future clinical application.

https://doi.org/10.1038/s41591-019-0745-3

\section{CANCER THERAPY}

\section{Chemotherapy signatures \\ Nat. Genet. 51, 1732-1740 (2019)}

Cancer therapies damage both cancerous tissues and healthy tissues, and the mutational signatures unique to each therapy informs understanding of their long-term side effects.

The DNA-damaging chemotherapies used as standard of

care for most cancers are highly toxic and have serious long-term and delayed side effects. However, the burden of mutation induced by each therapy and how these unique signatures might influence adverse effects have not yet been explored in detail.

A group of European researchers analyzed data from 3,506 metastatic tumors treated with six anticancer agents: five chemotherapies and one radiotherapy. They developed a new analytical approach for identifying mutational signatures attributable to specific therapies and were able to predict the burden of these therapies, which in the future may be used to inform clinicians.
PUBLIC HEALTH

\section{A conjugate vaccine for typhoid} N. Engl. J. Med. 381, 2209-2218 (2019)

A conjugate vaccine for typhoid is immunogenic in children in a region in which typhoid is endemic.

Typhoid fever is a systemic febrile illness that disproportionately affects children and teenagers and is a major public-health challenge in low- and middle-income countries. Although two vaccines are currently available, vaccination has not been widely implemented due to low immunogenicity and challenges in delivery to young children.

A typhoid conjugate vaccine ( $\mathrm{TCV}$ ) has recently been developed with the intention of improving immunogenicity relative to that of previous vaccines. In a phase 3 trial in Kathmandu, Nepal, where typhoid is a major problem, 20,019 children were randomly assigned to receive TCV or the MenA vaccine. A single dose of the TCV was immunogenic and was effective in reducing Salmonella Typhi bacteremia.
LIVER DISEASE

HS Initial promise for

NASH therapy

Lancet 394, 2184-2196 (2019)

A therapy targeting the liver farnesoid $\mathrm{X}$ receptor is showing promise for clinical benefit against non-alcoholic steatohepatitis (NASH).

NASH is a common cause of chronic liver disease, and its incidence is rising worldwide; however, the best current therapy is lifestyle intervention. Obeticholic acid is an agonist of the farnesoid $\mathrm{X}$ nuclear receptor that serves a role in the regulation of bile acids, and its activation is known to reduce hepatic fibrosis and inflammation, both of which are associated with the pathology of NASH.

A phase III clinical trial of obeticholic acid for NASH is ongoing in 320 centers in 20 countries across the world, including 1,968 patients. In an interim analysis, obeticholic acid significantly improved fibrosis and is expected to be of clinical benefit.

https://doi.org/10.1038/s41591-019-0742-6

\section{AUTOIMMUNE DISEASE \\ Common IBD pathogenic mechanisms \\ Cell 179, 1160-1176.e1 (2019)}

Pediatric-onset colitis and Crohn's disease share pathological mechanisms that may well be targetable.

Inflammatory bowel disease encompasses Crohn's disease and ulcerative colitis in children and substantially affects the development of those affected in childhood. Both are the result of a compromised intestinal mucosa; however, whether there is a shared pathogenesis of these conditions is unknown.

Huang et al. carried out single-cell sequencing and risk-gene analysis of children with undifferentiated colitis, Crohn's disease and ulcerative colitis. Their analysis identified a shared pathogenic mechanism that underlies mucosal immune defects. The mechanism is targetable, and in a pilot study of nine children treated with a therapeutic inhibitor, mucosal healing was promoted in the children.

https://doi.org/10.1038/s41591-019-0744-4 